\title{
VI Index des adjectifs-adverbes selon leur fréquence-type
}

Exemple: l'adjectif-adverbe fort se trouve dans 169 groupes syntaxiques différents, c'est-à-dire, il se combine avec 169 verbes différents

\begin{tabular}{|c|c|c|c|c|c|c|}
\hline fort & 169 & direct & 12 & calme & 4 & aimable \\
\hline haut & 145 & double & 12 & cru & 4 & amer \\
\hline bas & 120 & grave & 12 & loin & 4 & âpre \\
\hline droit & 110 & simple & 12 & positif & 4 & bouillant \\
\hline dur & 81 & chaud & 11 & pur & 4 & brut \\
\hline net & 78 & facile & 11 & sain & 4 & carré \\
\hline beau (bel) & 75 & franc & 11 & sucré & 4 & complet \\
\hline ferme & 73 & rapide & 11 & terrible & 4 & compliqué \\
\hline juste & 72 & vif & 11 & rond & 4 & comptant \\
\hline clair & 66 & gent & 10 & profond (parfont) & 4 & confortable \\
\hline menu & 58 & rouge & 10 & anglais & 3 & correct \\
\hline soef (suave) & 53 & utile & 10 & bizarre & 3 & courant \\
\hline $\mathrm{sec}$ & 51 & précis & 9 & démodé & 3 & dense \\
\hline court & 48 & seul & 9 & égal & 3 & destroit \\
\hline gros & 47 & seri (serein) & 9 & électrique & 3 & différent \\
\hline profond & 45 & creux & 8 & fier & 3 & douloureux \\
\hline dru & 42 & mauvais & 8 & gai & 3 & durable \\
\hline serré & 42 & plein & 8 & grief & 3 & économique \\
\hline petit & 40 & tranquille & 8 & joli & 3 & efficace \\
\hline faux & 32 & vert & 8 & joyeux & 3 & entier \\
\hline large & 30 & impec(cable) & 8 & lâche & 3 & figuratif \\
\hline long & 29 & aigu & 7 & local & 3 & flou \\
\hline doux & 27 & froid & 7 & mat & 3 & forcé \\
\hline raide & 27 & laid & 7 & moderne & 3 & fou \\
\hline étroit & 22 & propre & 7 & monotone & 3 & gavé \\
\hline cher & 20 & triste & 7 & mort & 3 & glacé \\
\hline blanc & 19 & vrai (voir) & 7 & neuf & 3 & global \\
\hline fin & 19 & bleu & 6 & pareil & 3 & gratuit \\
\hline léger & 19 & bref & 6 & pas cher & 3 & grêle \\
\hline bon & 18 & coi & 6 & pauvre & 3 & groseille \\
\hline vrai & 18 & jaune & 6 & ras & 3 & humide \\
\hline grand & 17 & maigre & 6 & riche & 3 & immobile \\
\hline noir & 17 & malin & 6 & rose & 3 & intelligent \\
\hline épais & 16 & moche & 6 & rude & 3 & inutile \\
\hline lourd & 16 & solide & 6 & sage & 3 & jovial \\
\hline gras & 15 & plat & 6 & sévère & 3 & leste \\
\hline isnel & 15 & chic & 5 & subit & 3 & libre \\
\hline frais & 14 & classique & 5 & sûr & 3 & mélancolique \\
\hline aise & 13 & prou & 5 & tendre & 3 & mortel \\
\hline lent & 13 & sérieux & 5 & vivant & 3 & moyen \\
\hline mou & 13 & bête & 4 & abondant & 2 & négatif \\
\hline
\end{tabular}




\begin{tabular}{|c|c|c|c|c|c|c|}
\hline niais & 2 & classe (class) & 1 & exact & 1 & mesquin \\
\hline ordonné & 2 & clos & 1 & excentrique & 1 & mi-long \\
\hline parfait & 2 & coco & 1 & extrême & 1 & mince \\
\hline poignant & 2 & cointe & 1 & fade & 1 & minuscule \\
\hline pointu & 2 & coloré & 1 & faible & 1 & misérable \\
\hline pratique & 2 & colossal & 1 & familier & 1 & modeste \\
\hline solidaire & 2 & compact & 1 & fanfaron & 1 & mollet \\
\hline sonore & 2 & compréhensible & 1 & fêlé & 1 & mollo \\
\hline souple & 2 & concentré & 1 & féminin & 1 & mordant \\
\hline tiède & 2 & concis & 1 & féroce & 1 & mou (mol) \\
\hline transparent & 2 & concret & 1 & figé & 1 & $\mathrm{mu}$ \\
\hline triple & 2 & confus & 1 & fixe & 1 & musclé \\
\hline vil & 2 & contemporain & 1 & flatteur & 1 & musical \\
\hline vulgaire & 2 & content & 1 & fluide & 1 & nerveux \\
\hline bleu-clair & 2 & contenu & 1 & fonctionnel & 1 & neutre \\
\hline nouveau (novel) & 2 & cosmique & 1 & frémissant & 1 & niquel \\
\hline abominable & 1 & couché & 1 & furtif & 1 & noble \\
\hline abstrait & 1 & coupé & 1 & gagnant & 1 & nonchalant \\
\hline acertes & 1 & courbe & 1 & gagné & 1 & normal \\
\hline âcre & 1 & critique & 1 & génial & 1 & nourrissant \\
\hline aérien & 1 & cuit & 1 & gothique & 1 & nu \\
\hline affectueux & 1 & décontracté & 1 & gourmand & 1 & obscur \\
\hline affranchi & 1 & déhanché & 1 & groupé & 1 & old school \\
\hline allongé & 1 & demi-creux & 1 & habillé & 1 & original \\
\hline ambigu & 1 & dépointé & 1 & hardi & 1 & osé \\
\hline ample & 1 & désespérant & 1 & herbal & 1 & ouvert \\
\hline amusant & 1 & détendu & 1 & heureux & 1 & paisible \\
\hline ancien & 1 & détroit & 1 & heurté & 1 & parallèle \\
\hline anguleux & 1 & difficile & 1 & honnête & 1 & penaud \\
\hline appétissant & 1 & digne & 1 & horizontal & 1 & penché \\
\hline appuyé & 1 & dynamique & 1 & idiot & 1 & perdant \\
\hline attractif & 1 & élégant & 1 & ignorant & 1 & personnel \\
\hline austère & 1 & enclos & 1 & immense & 1 & pesant \\
\hline automatique & 1 & engagé & 1 & imprévu & 1 & pimpant \\
\hline avenant & 1 & ennuyeux & 1 & incontinent & 1 & pire \\
\hline baroque & 1 & énorme & 1 & indépendant & 1 & pittoresque \\
\hline basique & 1 & enragé & 1 & indiscipliné & 1 & placé \\
\hline bio & 1 & enroué & 1 & infini & 1 & planqué \\
\hline blond & 1 & épicé & 1 & intact & 1 & poudré \\
\hline bon marché & 1 & équilibré & 1 & intellectuel & 1 & précaire \\
\hline branché & 1 & espacé & 1 & intime & 1 & précieux \\
\hline brouillé & 1 & estable & 1 & jeune & 1 & pressé \\
\hline brûlé & 1 & étendu & 1 & jus & 1 & profitant \\
\hline brusque & 1 & éternel & 1 & liquide & 1 & prudent \\
\hline brutal & 1 & éthéré & 1 & lisse & 1 & puant \\
\hline bruyant & 1 & étincelant & 1 & lumineux & 1 & puissant \\
\hline caché & 1 & étrange & 1 & machinal & 1 & quadruple \\
\hline campagnard & 1 & étriqué & 1 & malheureux & 1 & rade \\
\hline caverneux & 1 & évident & 1 & mélodieux & 1 & rangé \\
\hline
\end{tabular}




\begin{tabular}{|c|c|c|c|c|c|c|}
\hline rapidos & 1 & russe & 1 & souriant & 1 & tourmenté \\
\hline rare & 1 & safe (anglais) & 1 & soutenu & 1 & tranchant \\
\hline rasé & 1 & saignant & 1 & stable & 1 & triomphant \\
\hline rauque & 1 & salé & 1 & stratégique & 1 & tristos \\
\hline réaliste & 1 & satisfait & 1 & structuré & 1 & trouble \\
\hline réfléchi & 1 & savant & 1 & sublime & 1 & universel \\
\hline refroidi & 1 & savoureux & 1 & $\operatorname{succin}(c) t$ & 1 & végétal \\
\hline régulier & 1 & sensible & 1 & surgelé & 1 & vertueux \\
\hline renaissant & 1 & sexy & 1 & symétrique & 1 & vide \\
\hline rentré & 1 & sibilant & 1 & sympathique & 1 & vieux \\
\hline résolu & 1 & sobre & 1 & tapageur & 1 & vigoureux \\
\hline responsable & 1 & socialiste & 1 & tapi & 1 & violet \\
\hline retiré & 1 & soigné & 1 & tenace & 1 & virevoltant \\
\hline ridicule & 1 & solennel & 1 & terne & 1 & visuel \\
\hline rigide & 1 & sombre & 1 & tintant & 1 & volumineux \\
\hline rigolo & 1 & sourd & 1 & tortu & 1 & \\
\hline
\end{tabular}


